\title{
UM EXEMPLO DIDÁTICO PARA O ENSINO DA CONVOLUÇÃO DISCRETA
}

\section{A DIDATIC EXAMPLE FOR TEACHING DISCRETE CONVOLUTION}

\author{
Arian Ojeda González ${ }^{1}$ \\ Isabelle Cristine Pellegrini Lamin²
}

Resumo: Este artigo descreve um método didático para o ensino da convolução discreta. Através de um exemplo, apresenta-se o desenvolvimento matemático até definir a convolução discreta. Posteriormente, utilizando um segundo exemplo, estuda-se um método onde os termos do argumento da soma da convolução discreta são ordenados em uma tabela. Propõe-se que essa tabela irá facilitar o ensino de como calcular a convolução discreta sem usar o computador. O método tabelado é generalizado e transformado numa equação matricial sendo um caso especial de matriz de Toeplitz.

Palavras-chave: Convolução discreta; Matriz de Toeplitz; sistema linear Invariante ao deslocamento (LID).

Abstract: This article describes a didactic method for teaching discrete convolution. Using an example, the mathematical development is presented until discrete convolution is defined. Later, using a second example, a method is studied where the terms of the discrete convolution sum argument are ordered in a table. We propose that this table will facilitate the teaching of how to calculate discrete convolution without using the computer. The tabulated method is generalized and transformed into a matrix equation, corresponding to a special case of Toeplitz matrix.

Keywords: Discrete convolution; Toeplitz Matrix; linear time-invariant system (LTI).

\section{INTRODUÇÂO}

A palavra convolução origina-se do verbo convolar, este por sua vez assemelhase ao ato de "mudar de estado", em um sentido pouco conectado à matemática (mudar de estado civil, por exemplo, ou de partido político). De fato, na literatura não se encontra uma tradução direta do verbo "to convolve" para o português, eventualmente, detecta-se algum neologismo/anglicismo (tal como "convoluir" ou "convolucionar"). Linguisticamente não seria adequado considerar "convolução" como substantivo derivado de "convolar". O substantivo derivado, nesse caso, seria "convolação". No entanto, na literatura consultada em português (SPIEGEL, 1971; WALDMAN, 1987; ABRANTES, 2000; ZILL, 2003), utiliza-se o substantivo "convolução" para se referir à operação linear, que a partir de duas funções dadas, resulta numa terceira que mede a soma do produto dessas funções. Porém, não achamos adequado neste artigo substituir a palavra "convolução" por "convolação". Analogamente, em matemática, a convolução define-se como a modificação de um sinal de entrada para gerar um sinal de saída. Logo, essa transformação caracteriza um sistema linear de certo sinal, que seja invariante ao deslocamento. Dessa forma, verifica-se que o cálculo da convolução discreta é relevante para diversos estudos e áreas do conhecimento (YNOGUTI, 2017).

\footnotetext{
1 Docente e Pesquisador, Departamento de Física, IP\&D/Universidade do Vale do Paraíba-Univap, Brasil. Email: ojeda.gonzalez.a@gmail.com.

2 Graduação em andamento em Engenharia Civil, FEAU/Universidade do Vale do Paraíba-Univap, Brasil. E-mail: isa_lamin@hotmail.com.
} 
A convolução se relaciona com a integral de superposição na Óptica de Fourier, que por sua vez, descreve o sinal de saída de um Sistema Linear Invariante ao Deslocamento (LID) como a superposição ponderada das respostas ao impulso deslocadas no tempo (FIGUEIREDO, 1977; PEREIRA, 2011). A concepção de convolução está coesa com a análise e o processamento de sinais que são aplicados em várias áreas, dentre elas análise de imagens, como digitalização, alisamento, embasamento e aberração cromática, estatística, criptografia, acústica, oceanografia, sismologia, óptica, geometria, etc (ZILL, 2003; YNOGUTI, 2017).

Dessa forma, com base nessas noções, pode-se propor o objetivo deste trabalho, que se resume em uma nova forma didática de explicar a convolução discreta, a fim de auxiliar no sistema de ensino desta. A proposta de ensino apresentada neste trabalho surge como resultado da experiência de três anos (20142016) ministrando a disciplina de Processamento de Sinais na Universidade do Vale do Paraíba. Esta disciplina era oferecida como obrigatória para o curso de Engenharia da Computação e eletiva para os cursos: Engenharia Elétrica, Biomédica, Aeronáutica e Espaço. O pré-requisito exigido ao estudante é ter cursado as disciplinas de Cálculo I e II, Eletricidade Aplicada, Lógica de Programação e Métodos Numéricos. Porém, o método aplicado aqui, tem um enforque multidisciplinar que faria diferença e teria impacto positivo ao tentar aplicar o mesmo modelo em outras instituições de ensino.

Durante o desenvolvimento do artigo, abordam-se vários exemplos, e a partir deles, apresenta-se um método matricial para solucionar o cálculo da fórmula da convolução discreta. Apesar dos diversos autores (CARVALHO; VELOSO L; GURJÃO, 2015; VINAY et al., 2010; OPPENHEIM; SCHAFER; BUCK, 1999; HAYKIN; VAN VEEN, 2000) utilizarem outros métodos para aplicarem o conteúdo da convolução discreta, por meio de interpretações gráficas, equações e fórmulas, objetiva-se o intuito da aplicação de outros modos, menos utilizados, todavia, com mais facilidade de aprendizagem. Na prática, foi observado que, com a aplicação deste método, aproximadamente $90 \%$ dos alunos conseguiam resolver corretamente a pergunta na avaliação bimestral. Sendo que no ano de 2014 , com os outros métodos, a pergunta de convolução discreta foi resolvida corretamente por aproximadamente $60 \%$ dos estudantes.

\section{DEFINIÇÃO DA CONVOLUÇÃO DISCRETA}

O sinal impulso unitário discreto ou o sinal amostra unitária define-se como:

$$
\delta(n)=\left\{\begin{array}{lll}
1, & \text { se } & n=0 \\
0, & \text { se } & n \neq 0
\end{array}\right.
$$

Ele determina um único valor no instante $n=0$. Para deslocar o impulso unitário no tempo é preciso mudar o argumento da função $\delta(n)$ da seguinte forma, 


$$
\delta(n-2)=\left\{\begin{array}{lll}
1, & \text { se } & n=2 \\
0, & \text { se } & n \neq 2
\end{array}\right.
$$

Por exemplo, se $\mathbf{k}=\mathbf{2}$, a função desloca-se dois lugares à direita,

$$
\delta(n-k)=\left\{\begin{array}{lll}
1, & \text { se } & n=k \\
0, & \text { se } & n \neq k
\end{array}\right.
$$

Um sinal discreto $x(n)$ qualquer, pode ser representado como uma combinação linear dos impulsos unitários discretos deslocados no tempo (CARVALHO; VELOSO; GURJÃO, 2015). Para comprovar a ideia anterior, segue um exemplo.

\subsection{Exemplo 1}

Considerando o sinal $x(n)=\left\{1,-1,2, \frac{2}{\uparrow}, 5,4,3\right\},-3 \leq n \leq 3$, onde o símbolo $\uparrow$ indica o valor $x(0)$. Demonstre que:

$$
x(n)=\sum_{k=-\infty}^{+\infty} x(k) \cdot \delta(n-k) .
$$

Para demonstrar a igualdade anterior, primeiramente varia-se $\mathrm{k}$, no intervalo de 0 até 2, comprovando-se que:

$$
\begin{aligned}
x(n) \cdot \delta(n-0) & =x(0) \cdot \delta(n), \\
x(n) \cdot \delta(n-1) & =x(1) \cdot \delta(n-1), \\
x(n) \cdot \delta(n-2) & =x(2) \cdot \delta(n-2), \\
\ldots & \\
x(n) \cdot \delta(n-k) & =x(k) \cdot \delta(n-k) .
\end{aligned}
$$

Aplicando o somatório em k na Equação (5), têm-se:

$$
\sum_{k=-\infty}^{+\infty} x(n) \cdot \delta(n-k)=\sum_{k=-\infty}^{+\infty} x(k) \cdot \delta(n-k)
$$

Como x(n) não depende de k pode-se retirá-lo do somatório, como segue.

$$
x(n) \cdot \sum_{k=-\infty}^{+\infty} \delta(n-k)=\sum_{k=-\infty}^{+\infty} x(k) \cdot \delta(n-k) .
$$

Lembrando a relação $\sum_{k=-00}^{+\infty} \delta(n-k)=1$, conclui-se que:

$$
x(n)=\sum_{k=-\infty}^{+\infty} x(k) \cdot \delta(n-k) .
$$

Para continuar a análise, define-se o significado de um sistema de tempo discreto, na teoria de processamento de sinais. Conceitua-se um sistema como uma operação ou conjunto de operações que se realizam sobre um sinal de entrada $x(n)$ para produzir um sinal de saída $y(n)$. Ou seja, o sinal $x(n)$ é transformado pelo sistema em $y(n)$ (GRAY et al., 2006). Matematicamente, a relação entre $x(n)$ e $y(n)$ descreve-se da seguinte maneira: $y(n)=H[x(n)\rceil$ (ou $x(n) \rightarrow H \rightarrow y(n)$ ), onde o símbolo $\mathrm{H}$ representa 
o operador do sistema. Ou seja, o processo realizado pelo sistema $x(n)$ para produzir $y(n)$. No caso especial estudado neste trabalho, considera-se que o operador é um sistema linear invariante ao deslocamento (LID).

Na expressão matemática de $y(n)$ para um operador LID é substituído o termo $x(n)$ pela Equação (8):

$$
\begin{aligned}
& y(n)=L I D[x(n)], \\
& y(n)=L I D\left[\sum_{K=-\infty}^{+\infty} x(k) \cdot(\delta(n-k)] .\right.
\end{aligned}
$$

Conforme as propriedades do sistema linear invariante ao deslocamento, ele pode ser inserido dentro do somatório, como segue:

$$
y(n)=\sum_{k=-\infty}^{+\infty} x(k) \cdot \operatorname{LID}[\delta(n-k)]
$$

O termo $L I D[\delta(n-k)]$ é equivalente a $\delta(n-k) \rightarrow L I D \rightarrow h(n-k)$, cuja saída do sistema chama-se resposta ao impulso. Substituindo-se $h(n-k)$ em (10), define-se a convolução discreta denotada pelo símbolo do produto “*”, ou seja:

$$
y(n)=x(n) * h(n-k)=\sum_{k=-\infty}^{+\infty} x(k) \cdot h(n-k) .
$$

A definição da Equação (11) pode-se encontrar em qualquer livro de processamento de sinais. No entanto, foi identificado que os alunos tinham dificuldades no uso da formula da convolução discreta para aplicá-la em exercícios.

\section{UM MÉTODO ANALÍTICO PARA UTILIZAR A CONVOLUÇÃO DISCRETA}

Na literatura consultada (CARVALHO; VELOSO; GURJÃO, 2015; VINAY et al., 2010; OPPENHEIM; SCHAFER; BUCK, 1999) foi verificado que se utiliza apenas um método gráfico para explicar a convolução discreta. Nesta seção, apresenta-se um método matricial para facilitar a aprendizagem desse conteúdo. Um exemplo será utilizado como apoio à aprendizagem do método.

\subsection{Exemplo 2}

A resposta impulsiva de um sistema $L I D$ é $h(n)=\left\{1, \frac{2}{\uparrow}, 1,-1\right\},-1 \leq n \leq 2$. Determine a resposta $y(n)$ do sistema ao sinal de entrada $x(n)=\left\{\frac{1}{\uparrow}, 2,3,1\right\}, 0 \leq n \leq 3$. $\mathrm{O}$ primeiro passo é definir o intervalo $k$ dos limites do operador somatório $\left(\sum\right)$ da Equação (11) da soma de convolução. O produto $x(k) \cdot h(n-k)$ terá valores iguais a zero fora de um intervalo, por isso, para diminuir os cálculos o ideal será definir um intervalo que exclua os produtos iguais a zero, e inclua os restantes. Um método funcional é eleger o maior valor absoluto dos intervalos onde foram definidos $h(n) \mathrm{e}$ $x(n)$. Por exemplo, $h(n)$ foi definido no intervalo $-1 \leq n \leq 2$, porém, adotamos $n=2$. Em 
contrapartida $x(n)$ foi definido no intervalo $0 \leq n \leq 3$, no qual elegemos $n=3$. Ou seja, no final escolhemos o máximo entre $n=2$ e $n=3$, que nos permitirá definir um intervalo de $k$ (neste caso $-3 \leq k \leq 3$ ), no qual realizaremos os cálculos. Depois de determinados os limites podemos reescrever a fórmula da convolução como segue:

$$
y(n)=x(n) * h(n)=\sum_{k=-3}^{3} x(k) \cdot h(n-k) .
$$

O segundo passo consiste em calcular o valor de $y(n)$ quando $n=0$ :

$$
\begin{aligned}
y(0)= & \sum_{k=-3}^{3} x(k) \cdot h(0-k)=\cdots+0+x(-3) \cdot h(3)+x(-2) \cdot h(2)+x(-1) \cdot h(1)+ \\
& x(0) \cdot h(0)+x(1) \cdot h(-1)+x(2) \cdot h(-2)+x(3) \cdot h(-3)+0+\cdots
\end{aligned}
$$

Das informações fornecidas no enunciado do problema teremos que: $x(-3)=0, x(-2)=0, x(-1)=0, x(0)=1, x(1)=2, x(2)=3, x(3)=1$, $h(3)=0, h(2)=-1, h(1)=1, h(0)=2, h(-1)=1, h(-2)=0, h(-3)=0$. Logo, esses valores são substituídos na Equação (13) e o resultado será:

$$
\begin{aligned}
y(0)= & \cdots+0+0 \cdot 0+0 \cdot(-1)+0 \cdot 1+ \\
& 1 \cdot(2)+2 \cdot(1)+3 \cdot 0+1 \cdot 0+0+\cdots \\
= & 4 .
\end{aligned}
$$

Outra forma, mais prática, é colocar os valores de $k, x(k), h(0-k)$ e $x(k) \cdot h(0-k)$ numa tabela. Dessa forma, o somatório dos valores da última linha da tabela resultará em $y(0)$ como segue na Tabela 1 :

Tabela 1 - Valores de $k, x(k), h(0-k) \mathrm{e} x(k) \cdot h(0-k)$.

\begin{tabular}{lllllllll}
\hline$k:$ & -3 & -2 & -1 & 0 & 1 & 2 & 3 & \\
$x(k):$ & 0 & 0 & 0 & 1 & 2 & 3 & 1 & \\
$h(0-k):$ & 0 & -1 & 1 & 2 & 1 & 0 & 0 & \\
$x(k) \cdot h(0-k):$ & 0 & 0 & 0 & 2 & 2 & 0 & 0 & $\sum_{k=-3}^{3} x(k) \cdot h(0-k)=4$. \\
\hline
\end{tabular}

Fonte: Os autores.

O terceiro passo consiste em calcular o valor de $y(n)$ quando $n=1$ :

$$
\begin{aligned}
y(1)= & \sum_{k=-3}^{3} x(k) \cdot h(1-k)=\cdots+0+x(-3) \cdot h(4)+x(-2) \cdot h(3)+x(-1) \cdot h(2)+ \\
& x(0) \cdot h(1)+x(1) \cdot h(0)+x(2) \cdot h(-1)+x(3) \cdot h(-2)+0+\cdots
\end{aligned}
$$

Das informações fornecidas no enunciado do problema, teremos os valores que são substituídos na equação anterior. O resultado será:

$$
\begin{aligned}
y(1)= & \cdots+0+0 \cdot 0+0 \cdot 0+0 \cdot(-1)+ \\
& 1 \cdot(1)+2 \cdot(2)+3 \cdot 1+1 \cdot 0+\cdots \\
= & 8
\end{aligned}
$$

Prosseguindo com o mesmo raciocínio, os valores de $k, x(k), h(1-k) \mathrm{e}$ $x(k) \cdot h(1-k)$ também podem ser organizados em uma tabela. Se escolhermos de referência a tabela anterior, repetindo-se as duas primeiras linhas, movimentamos 
$h(-k)$ um valor à direita para obter $h(1-k)$, sendo assim, o resultado da última linha será $y(1)$ : como segue na Tabela 2:

Tabela 2 - Valores de $k, x(k), h(1-k)$ e $x(k) \cdot h(1-k)$.

\begin{tabular}{lllllllll}
\hline$k:$ & -3 & -2 & -1 & 0 & 1 & 2 & 3 & \\
$x(k):$ & 0 & 0 & 0 & 1 & 2 & 3 & 1 & \\
$h(1-k):$ & 0 & 0 & -1 & 1 & 2 & 1 & 0 & \\
$x(k) \cdot h(1-k):$ & 0 & 0 & 0 & 1 & 4 & 3 & 0 & $\sum_{k=-3}^{3} x(k) \cdot h(1-k)=8$. \\
\hline
\end{tabular}

Fonte: Os autores.

De acordo com os dois passos anteriores pode-se fazer uma única tabela para calcular todos os valores de $y(n)$ quando $0 \leq n \leq 6$ : como segue na Tabela 3

Tabela 3 - Valores de $y(n)$ para $h(n-k)$.

\begin{tabular}{llllllllll}
\hline & $k:$ & -3 & -2 & -1 & 0 & 1 & 2 & 3 & \\
& $x(k):$ & 0 & 0 & 0 & 1 & 2 & 3 & 1 & \\
\hline $\mathrm{n}$ & & & & & & & & \\
0 & $h(0-k):$ & 0 & -1 & 1 & 2 & 1 & 0 & 0 & $y(0)=4$ \\
1 & $h(1-k):$ & 0 & 0 & -1 & 1 & 2 & 1 & 0 & $y(1)=8$ \\
2 & $h(2-k):$ & 0 & 0 & 0 & -1 & 1 & 2 & 1 & $y(2)=8$ \\
3 & $h(3-k):$ & 0 & 0 & 0 & 0 & -1 & 1 & 2 & $y(3)=3$ \\
4 & $h(4-k):$ & 0 & 0 & 0 & 0 & 0 & -1 & 1 & $y(4)=-2$ \\
5 & $h(5-k):$ & 0 & 0 & 0 & 0 & 0 & 0 & -1 & $y(5)=-1$ \\
6 & $h(6-k):$ & 0 & 0 & 0 & 0 & 0 & 0 & 0 & $y(6)=0$. \\
\hline
\end{tabular}

Fonte: Os autores.

A partir de $n=6$, todos os $y(n)$ serão zeros e não é necessário continuar acrescentando linhas. Para calcular os $y(n)$ quando $n \leq-1$ utiliza-se um método similar, mas neste caso a partir de $h(0-k)$ movimentamos os $h(n-k)$ passo a passo para a esquerda, como segue na Tabela 4:

Tabela 4 - Valores de $y(n)$ quando $n \leq-1$ para $h(n-k)$

\begin{tabular}{llllllllll}
\hline & $k:$ & -3 & -2 & -1 & 0 & 1 & 2 & 3 & \\
& $x(k):$ & 0 & 0 & 0 & 1 & 2 & 3 & 1 & \\
\hline $\mathrm{n}$ & & & & & & & & & \\
0 & $h(0-k):$ & 0 & -1 & 1 & 2 & 1 & 0 & 0 & $y(0)=4$ \\
-1 & $h(-1-k):$ & -1 & 1 & 2 & 1 & 0 & 0 & 0 & $y(-1)=1$ \\
-2 & $h(-2-k):$ & 1 & 2 & 1 & 0 & 0 & 0 & 0 & $y(-2)=0$ \\
-3 & $h(-3-k):$ & 2 & 1 & 0 & 0 & 0 & 0 & 0 & $y(-3)=0$ \\
-4 & $h(-4-k):$ & 1 & 0 & 0 & 0 & 0 & 0 & 0 & $y(-4)=0$ \\
-5 & $h(-5-k):$ & 0 & 0 & 0 & 0 & 0 & 0 & 0 & $y(-5)=0$. \\
\hline
\end{tabular}

Fonte: Os autores.

Sendo que a solução final do exemplo será:

$$
y(n)=\left\{1, \frac{4}{\uparrow}, 8,8,3,-2,-1\right\},-1 \leq n \leq 5 .
$$

Outro modo de programar o método anterior será utilizando a Equação (11) em forma matricial. Logo, as duas tabelas anteriores podem ser escritas no formato 
matemático seguinte:

$$
H_{N \times K} \cdot X_{K \times 1}=Y_{N \times 1},
$$

sendo que $H_{N \times K}$ é uma matriz formada pelos valores de $h(n-k)$, enquanto que $X_{K \times 1}, Y_{N \times 1}$ são matrizes coluna cujos elementos são $x(k)$ e $y(n)$, respectivamente. A letra $N$ do índice da matriz é o número total de valores inteiros do intervalo $n$, e $K$ é o número total de valores inteiros no intervalo $k$. Supondo que $n=\{-2,-1, \ldots, 6\}$ e $k=\{-3,-2, \cdots, 3\}$, a Equação (11) será representada como segue:

$$
\left[\begin{array}{ccccccc}
h(1) & h(0) & h(-1) & h(-2) & h(-3) & h(-4) & h(-5) \\
h(2) & h(1) & h(0) & h(-1) & h(-2) & h(-3) & h(-4) \\
h(3) & h(2) & h(1) & h(0) & h(-1) & h(-2) & h(-3) \\
h(4) & h(3) & h(2) & h(1) & h(0) & h(-1) & h(-2) \\
h(5) & h(4) & h(3) & h(2) & h(1) & h(0) & h(-1) \\
h(6) & h(5) & h(4) & h(3) & h(2) & h(1) & h(0) \\
h(7) & h(6) & h(5) & h(4) & h(3) & h(2) & h(1) \\
h(8) & h(7) & h(6) & h(5) & h(4) & h(3) & h(2) \\
h(9) & h(8) & h(7) & h(6) & h(5) & h(4) & h(3)
\end{array}\right] \cdot\left[\begin{array}{c}
x(-3) \\
x(-2) \\
x(-1) \\
x(0) \\
x(1) \\
x(2) \\
x(3)
\end{array}\right]=\left[\begin{array}{c}
y(-2) \\
y(-1) \\
y(0) \\
y(1) \\
y(2) \\
y(3) \\
y(4) \\
y(5) \\
y(6)
\end{array}\right]
$$

Do ponto de vista matemático, existe uma lógica na hora de escrever as matrizes da equação anterior. A primeira coluna da matriz $H$ escreve-se com os valores de $h(1), h(2), \cdots h(N)$. Posteriormente, escrevem-se os termos de cada uma das próximas linhas a partir do primeiro valor de $h(n)$ da coluna à esquerda da mesma linha menos um. Por exemplo, sendo o valor da primeira coluna $h(1)$ na linha 1 , os termos da segunda coluna em diante serão na mesma linha: $h(1-1), h(1-2), h(1-3), \cdots, h(1-(K-1))$. Para melhor esclarecimento, na sexta linha o primeiro valor será $h(6)$ e os demais, $h(6-1), h(6-2), h(6-3), \cdots, h(6-(K-1))$. A matriz $H$ é uma matriz circulante, visto que cada linha é formada por um deslocamento cíclico de $i$ posições a direita, ou seja, que é um caso especial de matriz de Toeplitz (GRAY et al., 2006). Na álgebra linear, a matriz de Toeplitz, denominada assim em homenagem a Otto Toeplitz, é uma matriz quadrada, onde os elementos da sua diagonal (da esquerda à direita) são iguais. A matriz $X$ está constituída pelos elementos $x(k(1)), x(k(2)), \cdots, x(k(K))$. E por fim, a matriz $Y$ está composta pelos elementos $y(n(1)), y(n(2)), \cdots, y(n(N))$.

Dessa forma, o método apresentado poderá facilitar a aprendizagem do cálculo da convolução discreta, como definida pela Equação (18). Como comprovação do método utilizado, aplicam-se os valores do Exemplo 2 na fórmula matricial (19). Obtendo-se como resultado as matrizes a seguir: 


$$
\left[\begin{array}{ccccccc}
1 & 2 & 1 & 0 & 0 & 0 & 0 \\
-1 & 1 & 2 & 1 & 0 & 0 & 0 \\
0 & -1 & 1 & 2 & 1 & 0 & 0 \\
0 & 0 & -1 & 1 & 2 & 1 & 0 \\
0 & 0 & 0 & -1 & 1 & 2 & 1 \\
0 & 0 & 0 & 0 & -1 & 1 & 2 \\
0 & 0 & 0 & 0 & 0 & -1 & 1 \\
0 & 0 & 0 & 0 & 0 & 0 & -1 \\
0 & 0 & 0 & 0 & 0 & 0 & 0
\end{array}\right] \cdot\left[\begin{array}{l}
0 \\
0 \\
0 \\
1 \\
2 \\
3 \\
1
\end{array}\right]=\left[\begin{array}{c}
0 \\
1 \\
4 \\
8 \\
8 \\
3 \\
-2 \\
-1 \\
0
\end{array}\right]
$$

Verificando-se que obtemos a mesma solução representada na Equação (17).

\section{IMPLEMENTAÇÃO DA CONVOLUÇÃO DISCRETA UTILIZANDO O MATLAB}

Depois de apresentado o método matricial para o cálculo da Convolução Discreta, propõe-se a utilização do MATLAB para fazer um programa que lhes permita calcular qualquer exemplo relacionado ao sistema LID. Para a aplicação desse método, há duas formas, sem a utilização da função "Conv" nativa do MATLAB, e consequentemente, desenvolvimento de uma função para realizar a operação. Ou com a utilização do "Conv" para o cálculo. Para o primeiro caso, foi necessária a criação de uma função, dessa forma foram inseridas algumas funções auxiliares sugeridas por VINAY et al. (2010) em que se mostram os respectivos códigos em MATLAB para a efetivação do cálculo da Convolução Discreta, tais comandos são explicados a seguir:

- Sigfold: reversão no tempo de $h(k)$, ou seja, precisamos de $h(-k)$;

- Sigshift: deslocamento no tempo para obter todos os h(n-k);

- Sigmult: para obter multiplicação de sinais.

De acordo com essas funções, o algoritmo foi elaborado da seguinte forma:

$$
\text { function }[y n, k n]=\operatorname{Convolucao}(x, k 1, h, k 2) \text {. }
$$

O primeiro passo é definir o intervalo $k$ dos limites do operador soma da convolução, logo se tem:

$$
k=\max ([k 1, k 2]) \text {. }
$$

Em seguida, utiliza-se a função "Sigfold" para realizar a reversão no tempo de $h(k)$, ou seja, $h(-k)$, como segue:

$$
[h 0, k 11]=\operatorname{sigfold}(h, k 1)
$$

O terceiro passo consiste no cálculo $\mathrm{y}(\mathrm{n})$ para todos os $\mathrm{n}$ :

$$
\begin{aligned}
& y \boldsymbol{n}=[-2 * \boldsymbol{k}: 2 * \boldsymbol{k}] \\
& \boldsymbol{k n}=[-2 * \boldsymbol{k}: 2 * \boldsymbol{k}] \\
& \boldsymbol{l}=\mathbf{1} .
\end{aligned}
$$

Posteriormente, para se obter os $h(n-k)$ utiliza-se o deslocamento no tempo com a função $[y, n]=\operatorname{sigshift}(x, m, k)$, como segue:

$$
\text { for } \boldsymbol{i}=-2 * \boldsymbol{k}: 2 * \boldsymbol{k}
$$




$$
\begin{aligned}
& n=i \\
& {[h k, k 12]=\text { sigshift }(h 0, k 11, n) .}
\end{aligned}
$$

Por último, aplica-se $[y, n]=\operatorname{sigmult}(x 1, n 1, x 2, n 2)$ para obter a multiplicação de sinais:

$$
\begin{aligned}
& {[x k h k, k 22]=\operatorname{sigmult}(x, k 2, h k, k 12)} \\
& y n(l)=\operatorname{sum}(x k h k) \\
& k n(l)=i \\
& l=l+1
\end{aligned}
$$

Depois de construída a função Convolução, ela pode ser utilizada para resolver o exemplo 2 da seção 3.1 desse artigo, para isso tem-se que:

$$
\begin{aligned}
& h=[1,2,1,-1] ; k 1=[-1: 2] ; \\
& x=[1,2,3,1] ; k 2=[0: 3] ; \\
& {[y n, k n]=\text { Convolucao }(x, k 1, h, k 2) ;}
\end{aligned}
$$

Sendo que o gráfico do sinal de entrada $x(n)=1,2,3,1,0<n<3$ é o seguinte:

$$
\begin{aligned}
& \text { subplot }(3,1,1) ; \\
& \text { stem }(k 2, x) ; \\
& \text { title('Sequencia de } \left.x(n)^{\prime}\right) \\
& \text { axis }(-77-39) ; \\
& \text { xlabel('n'); } \\
& \text { ylabel(' } \left.x(n)^{\prime}\right) ;
\end{aligned}
$$

O gráfico da resposta impulsiva $h(n)=[1,2,1,-1]$, com $-1<n<2$ pode-se obter pelos comandos:

$$
\begin{aligned}
& \text { subplot }(3,1,2) ; \\
& \text { stem }(k 1, h) ; \\
& \text { title('Sequencia de } h[n] ') \\
& \text { axis }(-77-39) ; \\
& \text { xlabel('n'); } \\
& \text { ylabel('h(n)'); }
\end{aligned}
$$

E por último, o gráfico do sinal de saída y(n) versus kn é plotado a partir de:

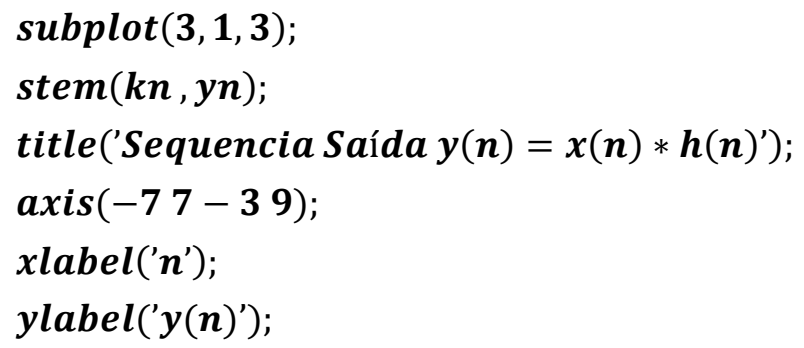

A Figura 1 mostra o resultado final do cálculo da convolução discreta a partir do exemplo anterior programado na linguagem MATLAB. 
Por outro lado, quando se utiliza a função "Conv" nativa do ambiente MATLAB, torna-se mais simples o cálculo da Convolução Discreta, visto que não há necessidade de criar uma função no código. Neste caso, é importante destacar que $h(k)$ e $x(k)$ devem ter o mesmo comprimento (ou seja, mesma quantidade de valores). Sendo necessário preencher com zeros aqueles valores que não são fornecidos no enunciado do exercício. Continuando com nosso exemplo, neste caso, as sequências de entrada serão da seguinte maneira:

$$
\begin{aligned}
& h=[0,0,1,2,1,-1,0] ; \\
& x=[0,0,0,1,2,3,1] ;
\end{aligned}
$$

Em seguida, chamamos a função convolução do MATLAB:

$$
\operatorname{conv}(\boldsymbol{h}, \boldsymbol{x}) \text {. }
$$

A resposta do programa sempre será uma sequência com comprimento ímpar, ou seja, o valor do meio da sequência corresponderá ao valor $n=0$ como segue:

$$
\text { ans }=[0,0,0,0,0,1,4,8,8,3,-2,-1,0],
$$

que corresponde aos seguintes valores de $n$ :

$$
n=[-6,-5,-4,-3,-2,-1,0,1,2,3,4,5,6] .
$$

Esses métodos contribuem para a compreensão do processamento de sinais e para se efetuar o cálculo da Convolução Discreta, tornando a aprendizagem mais dinâmica.

Figura 1 - Visualização da sequência de entrada $x(n)$, do filtro $h(n)$ e da saída $y(n)$ obtida a partir da fórmula da convolução discreta referentes ao exemplo 2 da seção 3.1. Os resultados foram visualizados a partir da implementação da função convolução apresentada na seção 4 .
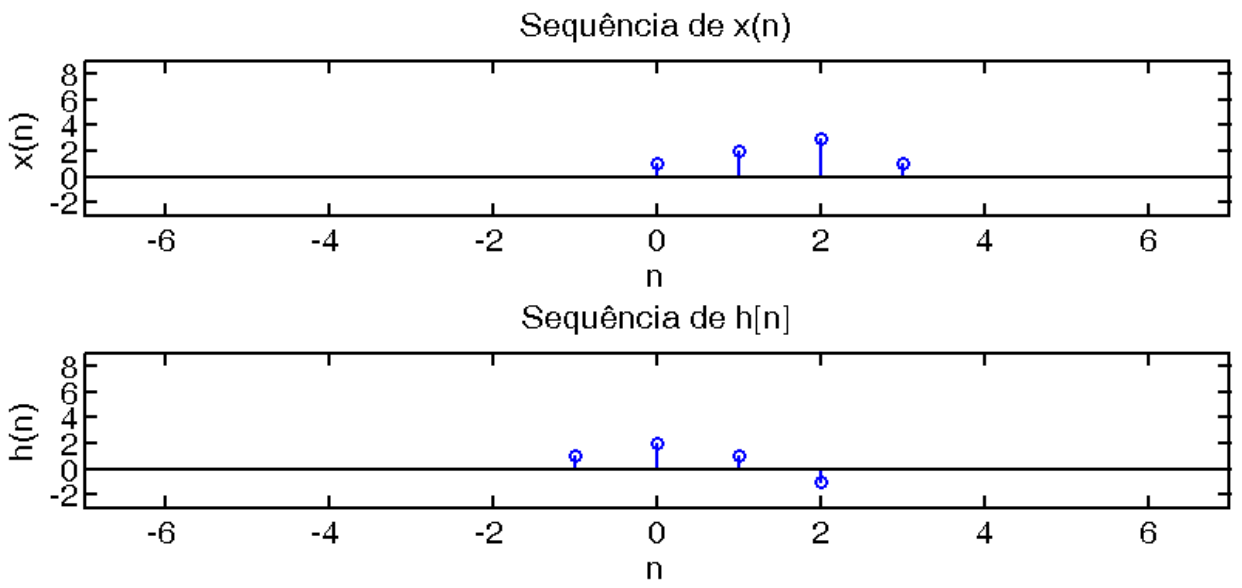

Sequência de Saída $y(n)=x(n)^{\star} h(n)$

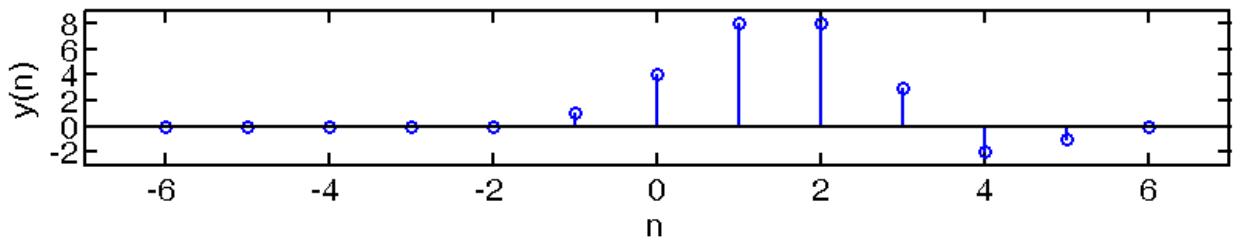

Fonte: Os autores. 


\section{CONSIDERAÇÕES FINAIS}

Neste artigo, mostra-se que o ensino da convolução discreta pode ser apresentado de forma matricial, pois através da abordagem estabelecida no decorrer do estudo, percebe-se que o intuito da análise do sistema linear invariante ao deslocamento- LID, refere-se a uma forma inovadora de aprendizagem deste cálculo, pautada nos conceitos de matrizes. Na literatura existente havia apenas ensino da convolução discreta por meio de fórmulas matemáticas, equações e gráficos. Dessa forma, o método desenvolvido neste artigo auxiliará no estudo desse processamento de sinal, de uma forma mais didática.

\section{AGRADECIMENTOS}

Em primeiro lugar, gostaríamos de agradecer ao Prof. Me. André Luiz de Toledo editor da Revista Univap pela ajuda durante o processo de revisão e edição do trabalho. Os autores também agradecem a dedicação dos revisores no processo de avaliação. Os autores agradecem ao CNPq (129109/2017-7; 800963/2016-4, 431396/2018-3) e a FAPESP (2018/02692-0) pelo apoio financeiro.

\section{REFERÊNCIAS}

ABRANTES, Sílvio A. Processamento Adaptativo de Sinais. Lisboa: Fundação Calouste Gulbenkian, 2000. 301 p.

CARVALHO, J. M.; VELOSO, L.; GURJÃO, E. C.. Análise de Sinais e Sistemas. Rio de Janeiro: Elsevier, 2015.

FIGUEIREDO, D. G. Análise de Fourier e Equações Diferenciais Parciais. 4. ed. Rio de Janeiro, RJ: Impa, 2000. 274p.

GRAY, R. M. Toeplitz and circulant matrices: A review. Foundations and Trends in Communications and Information Theory, v. 2, n. 3, 155-239, 2006.

HAYKIN, S. S.; VAN VEEN, B. Sinais e Sistemas. São Paulo: Bookman, 2001.

OPPENHEIM, A. V.; SCHAFER, R. W.; BUCK, J. R. Discrete Time Signal

Processing. 2. ed. New Jersey: Prentice Hall, 1999.

PEREIRA, A. G. Séries de Fourier e aplicações. Campina Grande: Universidade Estadual da Paraíba, 2011. Disponível em:

<http://dspace.bc.uepb.edu.br:8080/xmlui/handle/123456789/715>. Acesso em: 13 set, 2017.

SPIEGEL, Murray R. Transformadas de Laplace. São Paulo, SP: McGraw-Hill do Brasil, 1971. $344 \mathrm{p}$.

VINAY, K. et al. Digital Signal Processing using MatLab. 3. ed. Global Engineering: Christopher M. Shorttr, 2010. 
WALDMAN, Helio. Processamento Digital de Sinais: conceitos fundamentais. Buenos Aires: Kapelusz, 1987. 183p.

YNOGUTI, C. A. Processamento Digital de Sinais. Campus em Santa Rita do Sapucaí: Instituto Nacional de Telecomunicações, 2017. Disponível em:

<http://www.inatel.br/docentes/ynoguti/graduacao-sp-2113502489/52-convolucao>.

Acesso em: 13 set. 2017.

ZILL, D. G. Equações Diferenciais com Aplicações em Modelagem. São Paulo, SP: Thomson, 2003. xiv 492 p. 